\section{RNAi scores big wins}

November was a big month for Alnylam Pharmaceuticals' siRNA platform. On November 20, Alnylam gained FDA approval for Givlaari (givosiran), an aminolevulinate synthase inhibitor, for treating adults with acute hepatic porphyria. Four days later, Novartis swooped on The Medicines Company with a $\$ 9.7$ billion buyout offer, prompted by the progress of inclisiran, an siRNAbased inhibitor of proprotein convertase subtilisin/kexin type 9 (PCSK9), which The Medicines Company developed under license from Alnylam. Taken together, the two drugs are further evidence of the rapid maturation of the siRNA modality, which Alnylam has pioneered. With the cholesterollowering inclisiran, Novartis thinks it has a blockbuster drug on its hands, based on its estimate of 50 million patients in the main Western markets with excessively high low-density lipoprotein cholesterol (LDL-C) levels despite being on current standard-ofcare medications. Novartis has remained tight-lipped about pricing, but to gain widespread adoption inclisiran will need to cost far lower than the two approved antibody-based PCSK9 inhibitors, Sanofi/ Regeneron's Praluent (alirocumab) and Amgen's Repatha (evolocumab). Novartis has plenty of scope to undercut its rivals, given inclisiran's low and infrequent dose schedule, its smallmolecule-like production costs and its lack of cold-chain distribution or storage requirements. FDA approval is expected in late 2020. The economics of Givlaari are radically different from those of inclisiran, as it addresses a patient population of about 3,000 people in the United States and Europe. After discounts, it will cost about $\$ 442,000$ on an annual basis, but only if it delivers the same benefits observed in clinical trials, in which it reduced porphyria attacks by an average of $70 \%$ as compared with placebo. Givlaari represents Alnylam's second drug approval, after Onpattro (patisiran) for treating hereditary transthyretin-mediated amyloidosis in August 2018. Significantly, both Givlaari and inclisiran employ $\mathrm{N}$-acetylgalactosamine conjugation, a highly efficient liver-targeting delivery system. Targeting siRNA drugs to other organs remains a work in progress.

Published online: 9 January 2020 https://doi.org/10.1038/s41587-019-0384-8
All studies showed that persistent antigen stimulation induces TOX, which in turn mediates epigenetic changes that impose the exhausted condition. In the absence of TOX, no $\mathrm{T}_{\mathrm{EX}}$ cells form.

"When you conditionally delete TOX, you blunt the generation of $\mathrm{T}$ cells that have an exhausted phenotype," says Taylor Schreiber, CSO of Shattuck Labs, an immunotherapy company with a pipeline of checkpoint fusion proteins, who was not involved in the research.

Blocking TOX, therefore, could have therapeutic relevance. Anjana Rao, Patrick Hogan and their colleagues at the La Jolla Institute for Immunology (LJI) in California reported in one of the recent publications that CAR-T cells with reduced TOX function produce more cytokines, express fewer inhibitory receptors, display features associated with potent effector activity and show enhanced antitumor effects in mice.

TOX also interacts in a positive feedback loop with the transcription factor NR4A, making NR4A also of interest to targeting efforts. Both contribute to a network of transcription factors that pushes $\mathrm{CD} 8^{+} \mathrm{T}$ cells to an exhaustion phenotype. In earlier work in mice, the LJI researchers showed that when NR4A is eliminated it improves CAR-T cell efficacy against solid tumors. "That's a guideline for what you might expect in human patients," says Hogan, whose team is now collaborating with Lyell.

Wherry, for his part, demonstrated in his group's report that TCR-modified T cells with a single working copy of the TOX gene had superior tumor-fighting ability relative to cells with two. That finding, says Wherry, one of the scientific cofounders of ArsenalBio, suggests that TOX expression could be therapeutically adjusted in engineered cells to augment tumor control while still maintaining persistence and keeping runaway inflammation and autoimmunity in check. "You can actually strike this balance," he says.

Tamping down exhaustion is not always desirable, though. In September, a team co-led by immunologist Nicholas Haining, another ArsenalBio founder formerly of the Dana-Farber Cancer Institute in Boston, proposed another target, a phosphatase called PTPN2, involved in the immune cells' progression to exhaustion. The team reported that deleting Ptpn 2 helped shore up the number of fully exhausted T-cells without depleting the pool of progenitor exhausted $\mathrm{T}$ cells $\left(\mathrm{T}_{\mathrm{PEX}}\right)$, a population of stem-cell-like precursors with memory features that can both self-renew and give rise to terminally differentiated $\mathrm{T}_{\mathrm{EX}}$ cells. The upshot: bolstered antitumor immunity in a mouse model of melanoma.
"So, what that says is that you can have enhanced activity of T cells in a therapeutic and meaningful way without necessarily needing to shift the cells out of an exhausted state," says Haining, who now leads discovery oncology efforts at Merck Research Laboratories in Boston.

Independent support for the idea of targeting PTPN2 in adoptive T-cell therapies comes from the laboratory of Tony Tiganis, a biochemist at Monash University and the Peter MacCallum Cancer Centre in Melbourne, Australia, who has been studying PTPN2's role in $\mathrm{T}$ cells for more than a decade. In December, Tiganis and colleagues reported that inhibiting PTPN2 in T cells expressing a CAR specific for the oncoprotein HER-2 could eradicate cancer in mice bearing HER2 ${ }^{+}$mammary tumors. Overall, in mice at least, cells lacking in PTPN2 home better to tumor tissue, show enhanced activation after antigen engagement and are less prone to exhaustion. ArsenalBio and Tiganis are in talks about a potential research alliance.

The ultimate aim, however, is to use multiple approaches to engineer exhaustion resilience into T cells. ArsenalBio CSO Jane Grogan says the company plans to tap the collective know-how of its founders Alexander Marson and Kole Roybal from the University of California, San Francisco - experts in CRISPR-based genome engineering and synthetic biology circuit design, respectively. "Just having a one-tool approach won't get us a highly specific and maximally effective cell therapy product," she says.

Genetically expunging major drivers of exhaustion offers one route, but Lyell is also looking to overexpress transcription factors that activate $T$ cells and tip the regulatory scales away from any state of dysfunction. Late last year, Crystal Mackall, a Lyell scientific cofounder from Stanford University School of Medicine in California, reported that engineering CAR-T cells to overexpress an activation-related gene called JUN helps improve their expansion potential and antitumor activity. Thanks to the genetic jolt from $J U N$, which encodes a protein called c-Jun that is thought to inhibit other inducers of exhaustion, Mackall and her colleagues showed that the engineered cells showed enhanced potency in several mouse models. "There was an incredible transcriptional rewiring," Mackall says. "By overexpressing JUN, we can reset the balance to be more like a healthy cell."

ArsenalBio and Lyell now plan to advance cell therapies that are impervious to exhaustion in the first place. Yet the approach each company is taking is unlikely 